\title{
Variable effects of nicotine and anabasine on parasitized
}

\section{bumble bees [version 1; peer review: 2 approved with}

\section{reservations]}

\author{
Lukas P. Thorburn'1, Lynn S. Adler ${ }^{1}$, Rebecca E. Irwin²,3, Evan C. Palmer-Young ${ }^{1}$ \\ ${ }^{1}$ Department of Biology, University of Massachusetts at Amherst, Amherst, Massachusetts, USA \\ 2Department of Biology, Dartmouth College Hanover, New Hampshire, USA \\ ${ }^{3}$ Department of Applied Ecology, North Carolina State University, Raleigh, North Carolina, USA
}

\begin{tabular}{l} 
V1 First published: 21 Sep 2015, 4:880 \\
https://doi.org/10.12688/f1000research.6870.1 \\
Latest published: 16 Dec 2015, 4:880 \\
https://doi.org/10.12688/f1000research.6870.2 \\
\hline
\end{tabular}

\section{Abstract}

Secondary metabolites in floral nectar have been shown to reduce parasite load in two common bumble bee species. Previous studies on the effects of nectar secondary metabolites on parasitized bees have focused on single compounds in isolation; however, in nature, bees are simultaneously exposed to multiple compounds. We tested for synergistic effects of two alkaloids found in the nectar of Nicotiana spp. plants, nicotine and anabasine, on parasite load and mortality in bumble bees (Bombus impatiens) infected with the intestinal parasite Crithidia bombi. Adult worker bees inoculated with C. bombi were fed nicotine and anabasine diet treatments in a factorial design, resulting in four nectar treatment combinations: $2 \mathrm{ppm}$ nicotine, $5 \mathrm{ppm}$ anabasine, $2 \mathrm{ppm}$ nicotine and $5 \mathrm{ppm}$ anabasine together, or a control alkaloid-free solution. We conducted the experiment twice: first, with bees incubated under variable environmental conditions ('Variable'; temperatures varied from $10-35^{\circ} \mathrm{C}$ ); and second, under carefully controlled environmental conditions ('Controlled'; $27^{\circ} \mathrm{C}$ incubator, constant darkness). In 'Variable', each alkaloid alone significantly decreased parasite loads, but this effect was not realized with the alkaloids in combination, suggesting an antagonistic interaction. Nicotine but not anabasine significantly increased mortality, and the two compounds had no interactive effects on mortality. In 'Controlled', nicotine significantly increased parasite loads, the opposite of its effect in 'Variable'. While not significant, the relationship between anabasine and parasite loads was also positive. Interactive effects between the two alkaloids on parasite load were non-significant, but the pattern of antagonistic interaction was similar to that in the variable experiment. Neither alkaloid, nor their interaction, significantly affected mortality under controlled conditions. Our results do not indicate synergy between Nicotiana nectar alkaloids; however, they do suggest a complex interaction between secondary metabolites, parasites, and environmental variables, in which

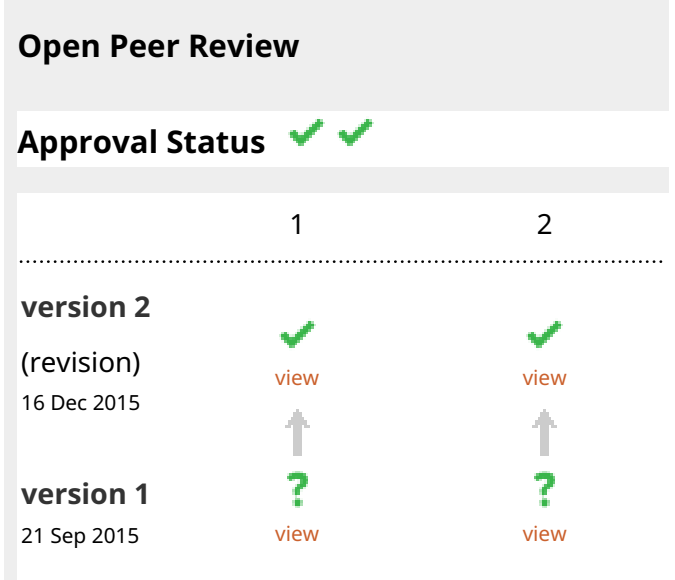

1. Michael Simone-Finstrom, USDA

Agricultural Research Service, Baton Rouge, USA

Margarita Lopez-Uribe, North Carolina State University, Raleigh, USA

Margarita Lopez-Uribe, North Carolina State University, Raleigh, USA

2. David Baracchi, Queen Mary, University of London, London, UK

Any reports and responses or comments on the article can be found at the end of the article. 
secondary metabolites can be either toxic or medicinal depending on context.

\section{Keywords}

Bumble bee, Bombus impatiens, parasites, Crithidia bombi , plant secondary metabolites, nicotine, alkaloids, tritrophic interactions

Corresponding author: Evan C. Palmer-Young (evan.palmeryoung@gmail.com)

Competing interests: No competing interests were disclosed.

Grant information: This research was funded by the National Science Foundation under NSFDEB-1258096, NSF GRFP (Grant DGE0907995 to ECPY), and NSF DDIG (Grant NSFDEB-1501907 to ECPY and LSA); by the National Research Initiative (NRI) Arthropod and Nematode Biology and Management Program of the USDA Cooperative State Research, Education, and Extension Service (CSREES) Grant no. USDA-AFRI 2013-02536; and by the Garden Club of America Centennial Pollinator Fellowship (ECPY). The funders had no role in study design, data collection and analysis, decision to publish, or preparation of the manuscript.

Copyright: @ 2015 Thorburn LP et al. This is an open access article distributed under the terms of the Creative Commons Attribution License, which permits unrestricted use, distribution, and reproduction in any medium, provided the original work is properly cited. Data associated with the article are available under the terms of the Creative Commons Zero "No rights reserved" data waiver (CC0 1.0 Public domain dedication).

How to cite this article: Thorburn LP, Adler LS, Irwin RE and Palmer-Young EC. Variable effects of nicotine and anabasine on parasitized bumble bees [version 1; peer review: 2 approved with reservations] F1000Research 2015, 4:880

https://doi.org/10.12688/f1000research.6870.1

First published: 21 Sep 2015, 4:880 https://doi.org/10.12688/f1000research.6870.1 


\section{Introduction}

Throughout the past two decades, many wild and managed bee species have experienced severe declines (Allen-Wardell et al., 1998; Cameron et al., 2011; Potts et al., 2010). In many cases of bee decline, parasitism has been implicated as a potential cause (reviewed in Goulson et al., 2015 and Potts et al., 2010). The parasitic mite Varroa destructor has been linked to honey bee declines in Ontario, Canada (Guzmán-Novoa et al., 2010). Infection with the microsporidian pathogen Nosema ceranae may be responsible for cases of honey bee colony collapse in Spain (Higes et al., 2009). Bumble bee species that have experienced recent declines had significantly higher levels of Nosema bombi than the species whose populations were stable (Cameron et al., 2011). Finding means of mitigating the effects of parasitism on bees would therefore be beneficial to the agricultural community as well as natural ecosystems.

Secondary metabolites - plant compounds that do not play a role in the plant's primary metabolism - frequently have antimicrobial properties (Schmidt et al., 2012), and could offer a means of natural parasite control. Secondary metabolites are found in the floral nectar of many plant species (Heil, 2011). The effects of secondary metabolites on insects, including bees and other pollinators, are context-dependent. A wide range of secondary metabolites, including terpenes, alkaloids, and phenolics, are toxic to insects (Detzel \& Wink, 1993; Kumrungsee et al., 2014; Raffa et al., 1985; Singaravelan et al., 2006; Wink \& Theile, 2002). Interaction with other stressors, such as infection or climatic stress, can exacerbate these toxic effects (Goulson et al., 2015; Holmstrup et al., 2010; Köhler et al., 2012a). However, under some circumstances, the antimicrobial properties of secondary metabolites can provide health benefits to infected insects. Insects have been shown to selfmedicate with secondary metabolites in response to parasite infection (reviewed in Abbott, 2014). For example, Grammia incorrupta (wooly bear) caterpillars exhibited self-medication behavior in response to tachinid fly parasitism by increasing their consumption of pyrrolizidine alkaloids, which decreased the survival of unparasitized caterpillars but increased the survival of parasitized caterpillars (Singer et al., 2009). Several recent studies have indicated that plant secondary metabolites, including those found in nectar, can benefit infected pollinators as well. Honey bees self-medicated in response to parasitism through increased foraging for resins, which are used in hive construction and have antimicrobial properties (SimoneFinstrom \& Spivak, 2012), and through preferentially feeding on certain types of honey, such as sunflower honey, which reduced pathogen load (Gherman et al., 2014). Consumption of the alkaloid gelsemine significantly reduced infection intensity in bumble bees (Bombus impatiens) infected with the intestinal parasite Crithidia bombi (Manson et al., 2010), and four other nectar secondary compounds had significant medicinal effects in the same bee-parasite system, with an additional four compounds causing non-significant decreases in infection severity (Richardson et al., 2015a).

Previous studies of the effects of nectar secondary metabolites on pollinators have focused primarily on single compounds in isolation. Under natural conditions, however, pollinators would likely encounter several compounds at once, since many plant species produce multiple secondary metabolites. For example, many Nicotiana species contain both nicotine and anabasine in nectar (Adler et al., 2012), and Chelone glabra contains the iridoid glycocides aucubin and catalpol in nectar (Richardson et al., 2015b). This raises the possibility of interactions between secondary metabolites in nectar. Synergistic interactions between secondary metabolites from other plant tissues are well established. The iridoid glycosides aucubin and catalpol had synergistic effects on the survival of common buckeye (Junonia coenia Hübner) caterpillars that specialize on plants with these compounds; caterpillars that consumed both iridoid glycosides had an increased rate of survival relative to caterpillars that consumed either glycoside alone (Richards et al., 2012). Amides in plants in the Piper genus had synergistic deterrent effects on herbivorous ants, while the same compounds were neutral or attractive in isolation (Dyer et al., 2003). Synergy between secondary metabolites can also alter antimicrobial effects. Carvacrol and thymol, for example, inhibited the growth of the bacterium Listeria innocua more effectively in combination than alone (García-García et al., 2011). Carvacrol was also more effective against the bacterium Vibrio cholerae when combined with cymene, although cymene alone had no antimicrobial activity (Rattanachaikunsopon \& Phumkhachorn, 2010). Antagonism between secondary metabolites has also been demonstrated. The deterrent effect of the amide piperine on the hemipteran Sibaria englemani is significantly reduced when piperine is combined with the amide piplartine, although piplartine alone had no effect on S. englemani feeding preference (Whitehead \& Bowers, 2014). The linear furanocoumarins psoralen, bergapten, and xanthotoxin exhibited antagonistic interactions in their effects on insect mortality; the toxicity of psoralen combined with either or both of the other two compounds was significantly lower than would be predicted based on their toxicities in isolation (Diawara et al., 1993). If similar interactions, either synergistic or antagonistic, are present between secondary metabolites in nectar, they could exacerbate or ameliorate the effects of single compounds found in previous studies.

To evaluate interactions between secondary metabolites from the nectar of a single plant, we tested the effects of nicotine and anabasine alone and in combination on bumble bee resistance to the gut parasite Crithidia bombi. Nicotine and anabasine co-occur in the nectar of several species in the genus Nicotiana, which includes cultivated tobacco (Nicotiana tabacum) as well as several ornamental species (Adler et al., 2012). The effects of nicotine and anabasine in combination on bee disease have not previously been studied.

We tested the effects of these compounds in two environmental contexts, variable and controlled conditions. Bumble bees in the wild encounter a wide range of environmental conditions, which could alter the effects of diet and parasitism. In general, temperature can decrease tolerance to environmental toxins, including secondary metabolites (Holmstrup et al., 2010), and exert unpredictable effects on insect-parasite interactions through modulation of host survival, host immune function, and parasite viability (Thomas \& Blanford, 2003). Variable temperatures impose exceptional energetic costs on bumble bees by forcing them to actively regulate body temperature in order to fly (Heinrich, 1972). These costs might create caloric deficits that increase parasite virulence in Bomubs (Brown et al., 2000). Alternatively, heightened energy needs could lead to increased consumption of plant foods, thereby elevating exposure to secondary metabolites. Globally, responses to environmental variability have implications for conservation: Bumble bee species with narrow climatic ranges are particularly 
vulnerable to decline (Williams et al., 2007; Williams et al., 2009), and projected climate change may further restrict these species' distributions through increases in mean temperature and the frequency of extreme events (Diffenbaugh \& Field, 2013).

\section{Methods}

Study system

Bombus impatiens is the most common bumble bee species in eastern North America, with a range extending from Ontario and Maine to southern Florida (Balaban et al., 2014). It is an important pollinator in agriculture, and commercial distribution of B. impatiens is becoming increasingly common (Colla et al., 2006).

Crithidia bombi is a common trypanosome parasite of bumble bees in Europe and North America (Colla et al., 2006; Lipa \& Triggiani, 1988). Its range has been expanding within North America and into parts of South America, potentially due to spillover from commercial to wild bumble bee populations (Colla et al., 2006; SchmidHempel et al., 2014; but see Whitehorn et al., 2013). C. bombi is known to increase mortality in bumble bees under food stress conditions (Brown et al., 2000), and to reduce bumble bee foraging rate (Otterstatter \& Thomson, 2006).

Nicotine is an agonist of the nicotinic acetylcholine receptor (nAChR), and therefore acts as both a stimulant drug and a toxin to many organisms (Benowitz, 1998). Nicotine is toxic to many insects, and has been historically used as an insecticide (Ujváry, 1999). Honey bees are deterred by nicotine in nectar (Köhler et al., 2012b), and both honey bees (Köhler et al., 2012b; Singaravelan et al., 2006) and bumble bees (Baracchi et al., 2015) are adversely affected by nicotine consumption when they are not infected by parasites. However, nicotine also has antimicrobial properties (Pavia et al., 2000), and recent studies have suggested that it can reduce parasite load in bumble bees infected with C. bombi (Baracchi et al., 2015; Richardson et al., 2015a), and may improve survival of diseased honey bee colonies (Köhler et al., 2012b). Anabasine, like nicotine, is a nAChR agonist, and has been used as an insecticide (MacBean, 2012). The behavioral effects of anabasine are similar to those of nicotine, although anabasine, unlike nicotine, does not have addictive effects (Caine et al., 2014). Anabasine in nectar has been found to deter honey bees (Singaravelan et al., 2005), and reduced C. bombi load in infected bumble bees (Richardson et al., 2015a).

\section{Diet treatments}

We inoculated bumble bees with $C$. bombi, and assessed the differences in pathogen load and mortality between adult bees fed nicotine (yes/no) and anabasine (yes/no) in a factorial design, resulting in four diet treatments: $2 \mathrm{ppm}$ nicotine, $5 \mathrm{ppm}$ anabasine, $2 \mathrm{ppm}$ nicotine and $5 \mathrm{ppm}$ anabasine together, or a control alkaloid-free solution. All diet treatments also contained $30 \%$ sucrose in distilled water. Chemicals ((-)-nicotine, cat. no. N3876; (+/-)-anabasine, cat. no. 284599) were purchased from Sigma-Aldrich (St. Louis, MO). Alkaloid concentrations were chosen to mimic the highest concentrations that would be found in Nicotiana nectar under natural conditions (Adler et al., 2006; Tadmor-Melamed et al., 2004).

\section{Rearing conditions}

We conducted two experiments. The first experiment ('Variable', conducted 26 February 2014 to 20 March 2014, Dataset 1) had a smaller sample size ( $\mathrm{n}=178$ bees) and less strictly controlled environmental conditions, while the second experiment ('Controlled', conducted 20 May 2014 to 14 July 2014, Dataset 2) had a larger sample size ( $\mathrm{n}=339$ bees) and carefully controlled environmental conditions (see sample sizes in Table S1). In 'Variable', experimental bees were kept on the lab bench (temperature range $10-35^{\circ} \mathrm{C}$ due to a steam leak, approximately $12 \mathrm{~h}$ photoperiod). In 'Controlled', experimental bees were incubated at $27^{\circ} \mathrm{C}$ in constant darkness to more closely mimic conditions in a bumble bee hive.

Experimental bees were obtained from pupal clumps of commercial B. impatiens (Biobest, Leamington, Ontario, Canada). Pupal clumps were removed from colonies weekly and kept in $500 \mathrm{~mL}$ plastic containers, with each container containing the pupal clumps from a single colony that were collected on a specific date. In 'Variable', pupal clumps were initially incubated on the lab bench, but were later incubated at $30^{\circ} \mathrm{C}$ in an incubator (Percival Scientific, Perry, IA) due to excessive pre-experiment mortality under the variable lab conditions. In 'Controlled', pupal clumps were incubated at $27^{\circ} \mathrm{C}$ throughout the experiment. Callow bees (newly emerged worker bees less than one day old) were collected upon emergence from pupal clumps. They were weighed and their mass at emergence, date of emergence, and colony of origin were recorded. Bees were assigned systematically to diet treatments in blocks of four, such that each block contained a bee in each treatment. Bees were then isolated in individual $20 \mathrm{~mL}$ vials. The lid of each vial was equipped with a $2 \mathrm{~mL}$ microcentrifuge tube with a cotton wick containing $500 \mu \mathrm{L}$ artificial nectar (30\% sucrose solution). Each day, bees were transferred to clean vials and given $500 \mu \mathrm{L}$ fresh artificial nectar and a $10 \mathrm{mg}$ piece of multifloral pollen (Koppert Biological Systems, Howell, MI) on which they fed ad libitum. For two days, bees were fed pollen and control nectar (30\% sucrose solution). Bees were inoculated with $C$. bombi two days after emergence. They were starved for several hours to ensure that they would consume the inoculum, and then fed $10 \mu \mathrm{L}$ of $C$. bombi inoculum containing 6,000 C. bombi cells (see below). Bees were then fed pollen and the appropriate nectar treatment ad libitum for 7 days.

\section{Inoculation}

To inoculate experimental bees, inoculum (C. bombi cells in sucrose solution) was prepared from the gut tracts of bees taken from colonies infected with $C$. bombi. These colonies were obtained from the same supplier as the experimental colonies, and were infected with $C$. bombi from wild bees collected in Amherst, Massachusetts (September 2013). Infected bees were dissected and their gut tracts were macerated with a plastic pestle in microcentrifuge tubes containing $300 \mu \mathrm{L}$ distilled water. Samples were incubated for 5 hours at room temperature to allow gut tissue to settle. C. bombi cell density was then assessed using a hemocytometer, and inoculum was prepared from the supernatant of the samples with sufficient concentrations of $C$. bombi cells. The supernatant was diluted to a concentration of 1200 cells/ $\mu \mathrm{L}$ and had an equal volume of $50 \%$ sucrose solution added to result in a $25 \%$ sucrose solution. Each bee was fed $10 \mu \mathrm{L}$ of inoculum, containing 6,000 C. bombi cells, using a $20 \mu \mathrm{L}$ micropipette.

Bumble bee dissection and parasite quantification

Seven days after inoculation, bees were dissected to assess parasite loads. Gut tracts were extracted and crushed with a pestle in 
microcentrifuge tubes containing $300 \mu \mathrm{L}$ distilled water. Samples were allowed to sit for 5 hours to allow gut tissue to settle. C. bombi cell concentrations in the gut extract were measured using a hemocytometer. C. bombi cells were counted in five cells of the hemocytometer and summed $(0.004 \mu \mathrm{L}$ each; $0.02 \mu \mathrm{L}$ total $)$.

\section{Statistics}

Data were analyzed using R version 3.2.1 for Windows (R Core Team, 2014).

\section{Mortality data}

For 'Variable', for which exact dates of death were not recorded, mortality was analyzed using a generalized linear mixed model with binomial error distribution (Pinheiro et al., 2015). Probability of death was used as the response variable with nicotine treatment, anabasine treatment, and their interaction as predictor variables. Bee colony was included as a fixed predictor, and date of inoculation was included as a random factor. Wald tests (Lesnoff \& Lancelot, 2012) were used to test the marginal significance of individual predictor variables (see Supplementary material script 1). Mortality data for 'Controlled', in which we recorded time from inoculation to death to the nearest day, were analyzed using a Cox proportional hazards mixed-effects model (Therneau, 2015). Death hazard rate was used as the response variable; nicotine, anabasine, and their interaction as predictor variables; colony as a fixed predictor; and date of inoculation as a random factor (see Supplementary material script 3).

\section{Parasite load}

Parasite counts were found to best fit the log-normal distribution and were analyzed using generalized linear mixed models (Bates et al., 2015) with penalized quasi-likelihood parameter estimation (Venables \& Ripley, 2002). Parasite counts were (x+1)-transformed for use as the response variable. Nicotine, anabasine, and their interaction were used as predictor variables. Bee colony was included as a fixed predictor, mass as a model covariate, and date of inoculation as a random factor. Marginal significance of individual terms was evaluated using Wald tests (Lesnoff \& Lancelot, 2012). Code for analysis is given in Supplementary material script 2 ('Variable' experiment) and Supplementary material script 4 ('Controlled' experiment).

\section{Results}

\section{Dataset 1. Data for 'Variable' experiment}

http://dx.doi.org/10.5256/f1000research.6870.d101937

Abbreviations: bee.ID—unique number assigned to each experimental bee; source.colony - colony of origin; treatmentletter corresponding to one of four diet treatments: " $\mathrm{C}$ " = control, "N" = nicotine (2 ppm), "A"= anabasine (5 ppm), "together" = nicotine (2 ppm) with anabasine (5 ppm); Nicotine.treatmentbinary variable for diet treatment indicating " 0 " for no nicotine or "1" for 2 ppm nicotine; Anabasine.treatment-binary variable for diet treatment indicating "0" for no anabasine or " 1 " for 5 ppm anabasine; mass-mass of bee at time of emergence from pupal clump; inoculation. date - date of inoculation; inoculated-binary variable indicating whether bee was successfully inoculated ("1") or not ("0"); dead.before.dissection—binary variable indicating whether bee died ("1") or survived ("0") until the time of dissection at 7 days; dissection.count-number of $C$. bombi cells counted in $0.02 \mu \mathrm{L}$ gut extract.

\section{Dataset 2. Data for 'Controlled' experiment}

http://dx.doi.org/10.5256/f1000research.6870.d101940

Abbreviations: bee- - unique number assigned to each experimental bee; colony - colony of origin; treatment-describes one of four diet treatments: "Control" = control; "Nicotine" = nicotine (2 ppm), "Anabasine" = anabasine $(5 \mathrm{ppm})$, "Nic + Ana" = nicotine $(2 \mathrm{ppm})$ with anabasine (5 ppm); Inoc.Date - date of inoculation; Nicotinecolumn denoting whether nectar treatment contained ("Yes") or did not contain ("No") 2 ppm nicotine; Anabasine_column denoting whether nectar treatment contained ("Yes") or did not contain ("No") 5 ppm anabasine; nicotine - binary variable for diet treatment indicating " 0 " for no nicotine or " 1 " for 2 ppm nicotine; anabasine - binary variable for diet treatment indicating " 0 " for no anabasine or " 1 " for 5 ppm anabasine; mass - mass of bee at time of emergence from pupal clump; Time. To. Death—number of days from inoculation to death, with negative numbers denoting excluded bees that died before inoculation or escaped before dissection; Dead. Binary - binary variable indicating whether bee died ("1") or survived ("0") until the time of dissection at 7 days; count-number of $C$. bombi cells counted in $0.02 \mu \mathrm{L}$ gut extract.

\section{'Variable'}

In variable temperature conditions, the nicotine treatment significantly increased mortality (Table 1). Nearly half of bees fed nicotine-containing nectar died within 7 days of inoculation, which was nearly double the frequency of death in treatments without nicotine (Figure 1). Anabasine did not affect mortality, and there was no significant interaction between the two alkaloid treatments (Figure 1, Table 1).

Nicotine (linear model $\beta=-1.01 \pm 0.295$ standard error) and anabasine ( $\beta=-0.94 \pm 0.31$ S.E.) each significantly decreased parasite loads. However, nicotine and anabasine displayed antagonistic effects (Nicotine * Anabasine $\beta=1.96 \pm 0.44$ S.E.), such that bees consuming both alkaloids did not realize the medicinal effects of either compound (Figure 2, Table 2).

\section{'Controlled'}

Under controlled conditions $\left(27^{\circ} \mathrm{C}\right.$ with constant darkness), neither alkaloid nor their interaction significantly affected mortality (Figure 3, Table 3). However, nicotine significantly increased parasite loads $(\beta=0.28 \pm 0.12$ S.E., Table 4$)$, while the effects of anabasine $(\beta=0.20 \pm 0.12$ S.E. $)$ were also positive but not significant

\section{Table 1. Effects of nicotine and anabasine consumption on mortality in 'Variable' experiment. Table shows binomial mixed model results of $\chi 2$ tests for effects of predictor variables on probability of death during the $7 \mathrm{~d}$ experiment.}

\begin{tabular}{|l|c|c|c|}
\hline Source & $\chi \mathbf{2}$ & Df & P \\
\hline Nicotine & 4.1749 & 1 & 0.041 \\
\hline Anabasine & 0.0374 & 1 & 0.85 \\
\hline Nicotine*Anabasine & 0.0256 & 1 & 0.87 \\
\hline Colony & 0.911 & 4 & 0.92 \\
\hline
\end{tabular}




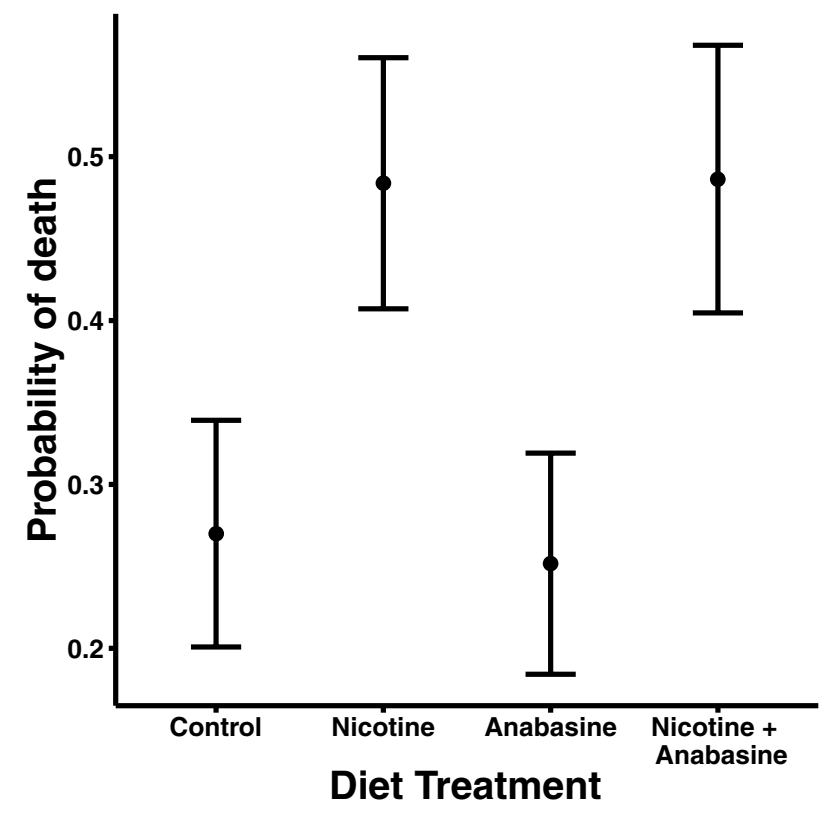

Figure 1. Effects of nicotine and anabasine on mortality in 'Variable' experiment. Points show adjusted mean probability of death in each treatment group. Error bars represent \pm 1 standard error.

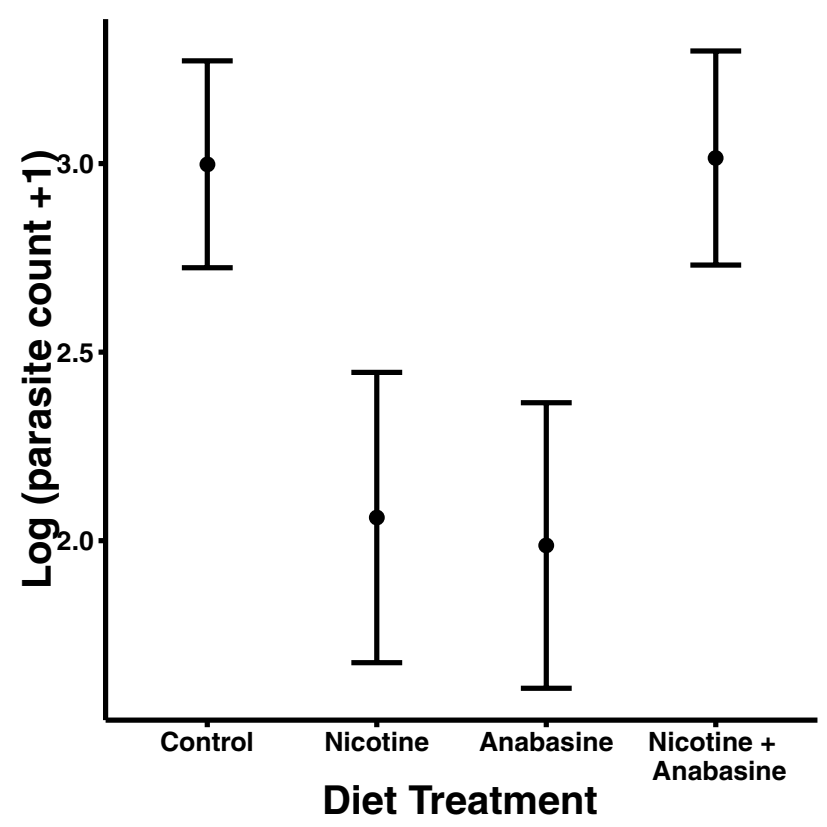

Figure 2. Effects of nicotine and anabasine on parasite load in 'Variable' experiment. Points show adjusted mean parasite count in each treatment group. Error bars represent \pm 1 standard error.
(Figure 4, Table 4). This was the opposite result of that observed in 'Variable', in which alkaloid ingestion decreased the severity of Crithidia infection. Although much weaker than in 'Variable', we found the same pattern of antagonistic interaction between the two alkaloids (Nicotine * Anabasine $\beta=-0.26 \pm 0.16$ S.E., Figure 4), indicating that the deleterious effects of each compound were reduced in bees consuming the nicotine-anabasine combination (Figure 4). However, this interaction was not statistically significant (Table 4). Overall parasite loads in 'Controlled' were much higher, with median parasite loads more than double those observed in 'Variable' (compare Figure 2 and Figure 4).

Table 2. Effects of nicotine and anabasine on parasite loads in 'Variable' experiment. Results of Wald tests for marginal significance of terms in a generalized linear mixed model with penalized quasi-likelihood parameter estimation.

\begin{tabular}{|l|c|c|c|}
\hline Source & $\boldsymbol{\chi 2}$ & Df & P \\
\hline Nicotine & 10.054 & 1 & 0.0025 \\
\hline Anabasine & 12.843 & 1 & $<0.001$ \\
\hline Nicotine*Anabasine & 22.045 & 1 & $<0.001$ \\
\hline Colony & 15.48 & 4 & 0.0038 \\
\hline Mass & 10.517 & 1 & 0.0012 \\
\hline
\end{tabular}

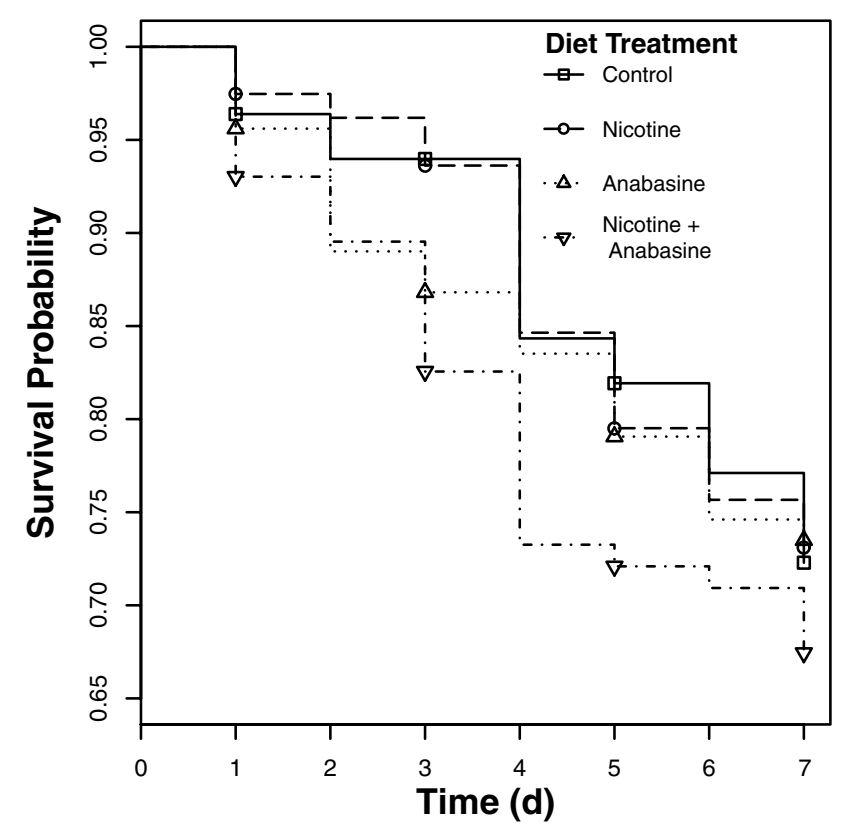

Figure 3. Effects of nicotine and anabasine on mortality in 'Controlled' experiment. Lines show survival curves for bees each treatment group. There were no significant effects of diet treatments on survival. 
Table 3. Effects of nicotine and anabasine consumption on mortality in 'Controlled' experiment. Table shows marginal significance of individual terms in Cox proportional hazards test for effects of predictor variables on mortality hazard rate.

\begin{tabular}{|l|c|c|c|}
\hline Source & $\boldsymbol{\chi 2}$ & $\mathbf{D f}$ & $\mathbf{P}$ \\
\hline Nicotine & 0.14 & 1 & 0.71 \\
\hline Anabasine & 0.21 & 1 & 0.65 \\
\hline Nicotine*Anabasine & 0.19 & 1 & 0.66 \\
\hline Colony & 7.6 & 3 & 0.054 \\
\hline
\end{tabular}

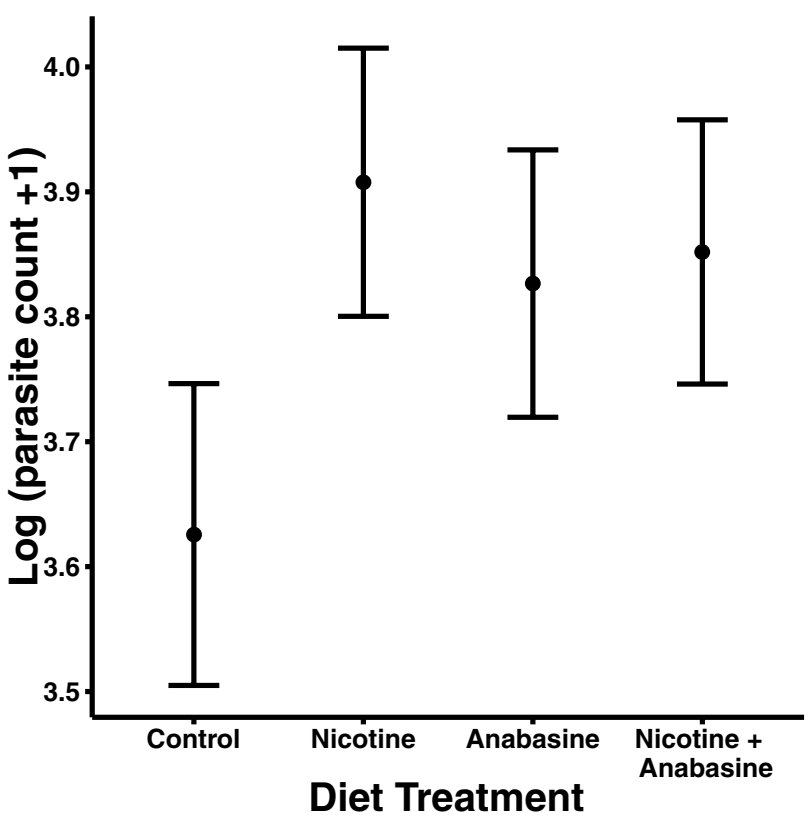

Figure 4. Effects of nicotine and anabasine on parasite load in 'Controlled' experiment. Points show adjusted mean parasite count in each treatment group. Error bars represent \pm 1 standard error.

Table 4. Effects of nicotine and anabasine on parasite loads in 'Controlled' experiment. Results of Wald tests for marginal significance of terms in a generalized linear mixed model with penalized quasi-likelihood parameter estimation

\begin{tabular}{|l|c|c|c|}
\hline Source & $\chi \mathbf{2}$ & $\mathbf{D f}$ & $\mathbf{P}$ \\
\hline Nicotine & 5.84 & 1 & 0.026 \\
\hline Anabasine & 2.78 & 1 & 0.095 \\
\hline Nicotine*Anabasine & 2.59 & 1 & 0.11 \\
\hline Colony & 6.76 & 3 & 0.080 \\
\hline Mass & 6.91 & 1 & 0.0086 \\
\hline
\end{tabular}

\section{Discussion}

Nicotine increased mortality under variable temperature conditions

Nicotine consumption increased mortality in 'Variable', but did not affect mortality in 'Controlled'. The difference in temperature between the two experiments may be responsible for this contextdependent response. In 'Variable', the incubation temperature of the experimental bees was not controlled. Bees were kept on lab benches, in a room with temperatures that ranged from 10 to $35^{\circ} \mathrm{C}$. In 'Controlled', by contrast, bees were incubated at a constant temperature of $27^{\circ} \mathrm{C}$. One hypothesis to explain these divergent responses is that bees in the first experiment may have experienced heat stress that could have exacerbated toxic effects of nicotine, and may have consumed larger quantities of the alkaloid-rich artificial nectar to compensate for evaporative water loss. However, we did not measure nectar consumption, and so cannot be certain that consumption increased under heat stress. Interaction between heat stress and secondary metabolites has been documented in several other species (reviewed in Holmstrup et al., 2010), including some insects and related arthropods. For example, Li et al. (2014) found synergistic interaction between heat stress and avermectin toxicity in the western flower thrips (Frankliniella occidentalis), which led to reduced survival and increased upregulation of heat shock proteins. Mercury exposure reduced heat tolerance in springtails (Folmosia candidia) (Slotsbo et al., 2009), and high temperature increased uptake and toxicity of organophosphate insecticides to the midge Chironomus tentans (Lydy et al., 1999). Our results suggest that interaction between heat stress and toxins may occur in B. impatiens as well. An experiment in which temperature and secondary metabolite consumption are manipulated in a factorial design would more definitively test for such interaction.

Our results indicate that nicotine can be toxic to bees even at very low concentrations when bees are parasitized. This contrasts with previous studies, which did not find significant toxic effects of nicotine at natural concentrations on mortality in bees of unknown parasite status. Detzel \& Wink (1993) determined the honey bee $\mathrm{LD}_{50}$ for nicotine to be $2000 \mathrm{ppm}$, far higher than any concentration that occurs in nectar. Singaravelan et al. (2006) found that larval survival of honey bees was not affected by naturally occurring concentrations of nicotine (up to $5 \mathrm{ppm}$ ), even when consumed consistently for several days, although a much higher concentration of nicotine $(50 \mathrm{ppm})$ did significantly reduce survival. These studies focused on honey bees, while our study used bumble bees, so the discrepancy between their results and ours may be due to bumble bees having a greater sensitivity to nicotine than honey bees. However, our results suggest that the toxic effects of nicotine are greater under temperature-stressed conditions; use of optimal incubation conditions could account for the lack of toxic effects observed in these previous studies. Drastic temperature variation similar to that experienced by bees in 'Variable' is common in continental climates. For example, in Amherst, MA, where this study was conducted, daily temperature swings of over $15^{\circ} \mathrm{C}$ are common, and temperatures as low as $10^{\circ} \mathrm{C}$ and as high as $30^{\circ} \mathrm{C}$ are frequently experienced within a few days of each other, or even within a single day (Menne et al., 2012a; Menne et al., 2012b). Wild bees, therefore, are likely to experience temperature conditions under which nicotine could be significantly toxic. 
Under variable temperature conditions, nicotine and anabasine-but not their combination-decreased infection

In 'Variable', bees that consumed either alkaloid alone had significantly lower parasite counts than control bees, but this effect was not present in bees that consumed both alkaloids. This is consistent with the results of recent studies that found reduced parasite loads under nicotine and anabasine consumption (Baracchi et al., 2015; Richardson et al., 2015a). The reduction in parasite load may be due to alkaloid-induced increases in gut motility. Both nicotine and anabasine have been demonstrated to reduce gut transit time in the Palestinian sunbird Nectarinia osea (Tadmor-Melamed et al., 2004). Although their effect on gut transit time in insects has not been studied, rapid excretion is known to be part of some insects' physiological response to alkaloids (Wink \& Theile, 2002). It is therefore plausible that consumption of nicotine and anabasine could cause an increased rate of excretion in bees, thus clearing C. bombi cells from the gut and leading to the observed reduction in parasite load.

The lack of effect of the combined alkaloids on parasite load is more puzzling. The concentrations of the individual alkaloids may have been within the medicinal window of concentration at which antiparasitic effects were dominant. However, the combined effects of both alkaloids may have weakened bees' ability to fight infection through excessive stimulatory, laxative, and/or immunosuppressive effects. These combined toxic effects could have offset the medicinal effects realized at lower concentrations in the single-alkaloid treatments.

In a controlled temperature environment, nicotine increased parasite loads without affecting mortality

In 'Controlled', nicotine consumption significantly increased parasite counts, while anabasine also increased parasite loads, although not significantly. This result is consistent with a growing body of research demonstrating that neonicotinoids, a class of insecticides chemically similar to nicotine, have immunosuppressant effects on bees (reviewed in Goulson et al., 2015). While the effects of nicotine are not necessarily the same as those of neonicotinoids, both nicotine and neonicotinoids function as nAChR agonists, (Jeschke et al., 2011), suggesting similar pharmacological activity. The immunosuppressant effects of neonicotinoids have been most well studied in honey bees. Sub-lethal colony-level exposure to the neonicotinoid imidacloprid has been shown to lead to increased levels of Nosema infection in honey bees (Pettis et al., 2012). A field study by Alburaki et al. (2015) found significantly higher levels of infection by both brood queen cell virus and Varroa mites in honey bee colonies that had foraged on corn treated with the neonicotinoid thiabendazole than in colonies that had foraged on untreated corn. The neonicotinoid pesticides clothianidin and imidacloprid induced increased transcription of a gene coding for a negative modulator of NF-K $\beta$ immune signaling in honey bees, causing decreased immune function and increased viral replication (Di Prisco et al., 2013).

Interactive effects of abiotic conditions, alkaloids, and parasites on bees

The apparent contradiction between the results of our first and second experiments may be due to a complex multi-directional interaction between alkaloid consumption, heat stress, and immunity. Under the variable conditions of 'Variable', bees may have consumed more liquid, causing them to ingest greater amounts of alkaloids. This increased alkaloid consumption could lead to stronger effects of the alkaloids, both in the form of increased toxicity and increased gut motility, accounting for both the higher mortality and decreased $C$. bombi counts in 'Variable'. Bees in 'Variable' were also exposed to external stimuli in the lab environment, including light and vibration, which may have further promoted alkaloid consumption by increasing energetic requirements and synergized with stimulatory effects of the alkaloids to promote intestinal peristalsis.

The higher temperatures of 'Variable' may have additionally functioned as an externally imposed fever that reversed the immunosuppressive effects of nicotine. Febrile amelioration of infection has been shown in many animals (reviewed in Kluger, 1978), including honey bees (Campbell et al., 2010) and other insects (Stahlschmidt \& Adamo, 2013). The lower absolute parasite counts relative to 'Controlled' may reflect heat-related inhibition of C. bombi, which grows best at $27^{\circ} \mathrm{C}$ (Salathé et al., 2012). Stimulatory effects of nicotine and anabasine, enhanced by exposure to everyday disturbance in 'Variable', could have increased activity level and metabolic rate, thereby further raising body temperature and slowing parasite growth. The effects of a given increase in body temperature would have been more pronounced under the hot conditions of 'Variable', which may have approached the parasite's thermal tolerance limit.

Our results contrast with the results of a recent study by Richardson et al. (2015a). Richardson et al., found that both nicotine and anabasine significantly reduced $C$. bombi parasite load in bumble bees without affecting mortality. In 'Variable', we similarly found that both alkaloids reduced pathogen load, but we also found that nicotine increased mortality. This discrepancy may be due to a simple difference in study design: we used the (-)-enantiomer of nicotine, whereas Richardson et al. (2015a) used a +/- enantiomeric mixture (Sigma N0267, personal communications). (-)-Nicotine is far more common in nature, comprising between $99.77 \%$ and $99.83 \%$ of the nicotine in tobacco (Armstrong et al., 1998), and is more pharmacologically active than (+)-nicotine. In vertebrates, (-)-nicotine was 2.4-3.1 times more toxic to vertebrates than was (+)-nicotine (Gause \& Smaragdova, 1939) and had stronger effects on the peripheral nervous system, particularly on muscle contraction (Barlow \& Hamilton, 1965). In aquatic invertebrates known to use acetylcholine as a neurotransmitter, (-)-nicotine was again on average 2.6-fold more toxic that the (+)-enantiomer (Gause \& Smaragdova, 1939). In insects, the (-)-enantiomer had stronger affinity for the nAChR in housefly and honey bee head membranes (Tomizawa \& Yamamoto, 1992). Interestingly, Gause \& Smaragdova (1939) found the two enantiomers to be isotoxic to the protozoans they tested. If this is the case for C. bombi, it suggests an explanation for our difference in results. If (-)-nicotine is more toxic to bees than (+)-nicotine, but both enantiomers are equally toxic to $C$. bombi, than (+)-nicotine could reduce parasite count without significantly affecting bee mortality, while (-)-nicotine could reduce parasite count but also be toxic to bees.

Another possible explanation for our differing results is a difference in the $C$. bombi itself. $C$. bombi is known to be genetically diverse; 
Salathé \& Schmid-Hempel (2011) identified 213 strains infecting bumble bees in Switzerland. Multiple strains are often present in a single host. Tognazzo et al. (2012) found that $67 \%$ of infected workers and 54\% of infected queens carried mixed-genotype infections, with queens harboring up to 29 different genotypes. In addition, it is possible that not all supposed $C$. bombi infections in fact represent a single Crithidia species. Schmid-Hempel \& Tognazzo (2010) identified two genetically and morphologically distinct lineages within the $C$. bombi complex, which they classified as cryptic species. They retained the name $C$. bombi for the lineage which more closely matches Lipa \& Triggiani's (1988) original description of C. bombi, and proposed the name $C$. expoeki for the other lineage. Both lineages are present in both Europe and North America, suggesting an old divergence. If our $C$. bombi cultures and those used by Richardson et al. (2015a) represent different strains, or different species, it is possible that they vary in their alkaloid tolerance.

\section{Implications of secondary metabolites for pollinator health} in a changing landscape

Our results represent an important first step towards understanding the interactive effects of multiple secondary metabolites on pollinators. We did not find evidence for synergy between Nicotiana nectar alkaloids, although we did find some evidence for antagonism. To elucidate the potential role of interactions between compounds in the plant-pollinator-parasite system, it will be necessary to test for interactions between other sets of compounds. Within Nicotiana, the wild tobacco $N$. attenuata contains at least 35 nectar secondary compounds, including sesquiterpenes (Kessler \& Baldwin, 2007); many terpenoids have potent trypanocidal activity, yet are relatively benign to animal cells (Otoguro et al., 2011). Among other plant families, Asclepias species are pollinated by bumble bees and contain several cardenolides in their nectar (Manson et al., 2012) that could be tested for interactive effects. Another plant species to investigate is Chelone glabra, which has high concentrations of the iridoid glycosides aucubin and catalpol in its nectar (Richardson et al., 2015b). Synergy between these glycosides has been demonstrated in their effect on Junonia coenia caterpillars (Richards et al., 2012).

The effect of nectar alkaloids on parasitized pollinators may represent a tradeoff between toxicity to the parasite and toxicity to the host. In the case of nicotine, bees appear to be more sensitive to alkaloid toxicity than parasites are. While nicotine inhibits the growth of many microbial pathogens, significant antimicrobial effects require concentrations between 100 and $250 \mathrm{ppm}$ (Pavia et al., 2000). By contrast, Singaravelan et al. (2006) found that nicotine was toxic to bees at $50 \mathrm{ppm}$, and our own results suggest that nicotine can have toxic effects at concentrations as low as $2 \mathrm{ppm}$. This suggests that bees are less tolerant of nicotine than microbes are. Despite their significant toxicity, nectar secondary metabolites such as nicotine are unlikely to pose a health risk to bees in the wild. The studies establishing toxicity of nicotine in bees have all focused on chronic consumption of a diet high in nicotine. This is an artificial condition that bees would be unlikely to encounter in nature. Bumble bees are generalist pollinators, and are known to forage on several plant species within a narrow time frame and even within a single foraging trip (Free, 1970). They would therefore be unlikely to consume enough nicotine from nectar to experience toxic effects, although exposure to pharmacologically similar, agriculturally ubiquitous neonicotinoid insecticides appears concerning (Goulson et al., 2015).

\section{Conclusion}

Our results emphasize the importance of interactions between stressors in pollinator health, and demonstrate that the effect of any single factor can vary greatly depending on the other factors involved. Research on pollinator health often focuses on single factors in isolation; however, in natural conditions, pollinators are often exposed to several stressors simultaneously (Goulson et al., 2015). Previous research has demonstrated both medicinal and toxic effects of secondary metabolites such as nicotine and anabasine. Our results suggest that the predominant effect can vary with environmental context. In order to better elucidate the role of secondary metabolites in pollinator health, future research should explicitly address the role of these complex interactions.

\section{Data availability \\ F1000Research: Dataset 1. Data for 'Variable' experiment, 10.5256/ f1000research.6870.d101937 (Thorburn et al., 2015a).}

F1000Research: Dataset 2. Data for 'Controlled' experiment, 10.5256/f1000research.6870.d101940 (Thorburn et al., 2015b).

\section{Author contributions}

All authors conceived the study. ECPY and LPT designed and conducted the experiments using methods developed by REI. ECPY analyzed the data. LPT and ECPY prepared the first draft of the manuscript. All authors revised the manuscript and have agreed upon the final content.

\section{Competing interests}

No competing interests were disclosed.

\section{Grant information}

This research was funded by the National Science Foundation under NSFDEB-1258096, NSF GRFP (Grant DGE-0907995 to ECPY), and NSF DDIG (Grant NSFDEB-1501907 to ECPY and LSA); by the National Research Initiative (NRI) Arthropod and Nematode Biology and Management Program of the USDA Cooperative State Research, Education, and Extension Service (CSREES) Grant no. USDA-AFRI 2013-02536; and by the Garden Club of America Centennial Pollinator Fellowship (ECPY).

I confirm that the funders had no role in study design, data collection and analysis, decision to publish, or preparation of the manuscript.

\section{Acknowledgments}

The authors are grateful to Biobest for supplying bee colonies; and to Ali Hogeboom, Melissa Ha, Caitlin McAllister, and lab assistants for help with 'Controlled' experiment. 


\section{Supplementary material}

Script 1. R script for analysis of C. bombi parasite loads in 'Variable' experiment.

Click here to access the data.

http://dx.doi.org/10.5256/f1000research.6870.s101933

Script 2. R script for analysis of mortality in 'Variable' experiment.

Click here to access the data.

http://dx.doi.org/10.5256/f1000research.6870.s101934

Script 3. R script for analysis of C. bombi parasite loads in 'Controlled' experiment.

Click here to access the data.

http://dx.doi.org/10.5256/f1000research.6870.s101935

Script 4. R script for analysis of mortality in 'Controlled' experiment.

Click here to access the data.

http://dx.doi.org/10.5256/f1000research.6870.s101936

Table S1. Sample sizes for the 2 experiments,

'Variable' and 'Controlled'.

\begin{tabular}{|l|r|r|}
\hline \multirow{2}{*}{ Treatment } & \multicolumn{2}{|c|}{ Experiment } \\
\hline Control & Variable & Controlled \\
\hline Nicotine & 45 & 83 \\
\hline Anabasine & 46 & 79 \\
\hline Nicotine + Anabasine & 46 & 91 \\
\hline Total & 41 & 86 \\
\hline
\end{tabular}

Abbott J: Self-medication in insects: current evidence and future perspectives. Ecol Entomol. 2014; 39(3): 273-280.

Publisher Full Text

Adler LS, Seifert MG, Wink M, et al:: Reliance on pollinators predicts defensive chemistry across tobacco species. Ecol Lett. 2012; 15(10): 1140-1148.

PubMed Abstract | Publisher Full Text

Adler LS, Wink M, Distl M, et al.: Leaf herbivory and nutrients increase nectar alkaloids. Ecol Lett. 2006; 9(8): 960-967.

Publed Abstract | Publisher FullText

Alburaki M, Boutin S, Mercier PL, et al:: Neonicotinoid-Coated Zea mays Seeds Indirectly Affect Honeybee Performance and Pathogen Susceptibility in Field Trials. PLoS One. 2015; 10(5): e0125790.

PubMed Abstract | Publisher Full Text | Free Full Text

Allen-Wardell G, Bernhardt P, Bitner R, et al:: The potential consequences of pollinator declines on the conservation of biodiversity and stability of food crop yields. Conserv Biol. 1998; 12(1): 8-17.

Publisher Full Text

Armstrong DW, Wang X, Ercal N: Enantiomeric composition of nicotine in smokeless tobacco, medicinal products, and commercial reagents. Chirality. 1998; 10(7): 587-591.

Publisher Full Text

Balaban J, Balaban J, Moisset B, et al.: Species Bombus impatiens - common eastern bumble bee. 2014

Reference Source

Baracchi D, Brown MJF, Chittka L: Behavioural evidence for self-medication in bumblebees? [version 2; referees: 3 approved]. F1000Res. 2015; 4: 73. Publisher Full Text

Barlow RB, Hamilton JT: The stereospecificity of nicotine. Br J Pharmacol Chemother. 1965; 25(1): 206-212.

PubMed Abstract | Publisher Full Text | Free Full Text 
Bates D, Maechler M, Bolker B, et al:: R package $\{$ Ime4\}: Linear mixed-effects models using \{Eigen\} and \{S4\}. 2015.

Reference Source

Benowitz NL: Nicotine safety and toxicity. Oxford University Press, New York, New York. 1998.

Reference Source

Brown MJF, Loosli R, Schmid-Hempel P: Condition-dependent expression of virulence in a trypanosome infecting bumblebees. Oikos. 2000; 91(3): 421-427. Publisher Full Text

Caine SB, Collins GT, Thomsen M, et al:: Nicotine-like behavioral effects of the minor tobacco alkaloids nornicotine, anabasine, and anatabine in male rodents. Exp Clin Psychopharmacol. 2014; 22(1): 9-22.

PubMed Abstract | Publisher Full Text

Cameron SA, Lozier JD, Strange JP, et al.: Patterns of widespread decline in North American bumble bees. Proc Natl Acad Sci U S A. 2011; 108(2): 662-667. PubMed Abstract | Publisher Full Text | Free Full Text

Campbell J, Kessler B, Mayack C, et al.: Behavioural fever in infected honeybees: parasitic manipulation or coincidental benefit? Parasitology. 2010; 137(10): 1487-1491.

PubMed Abstract | Publisher Full Text

Colla SR, Otterstatter MC, Gegear RJ, et al:: Plight of the bumble bee: Pathogen spillover from commercial to wild populations. Biol Conserv. 2006; 129(4): 461-467.

Publisher Full Text

Detzel A, Wink M: Attraction, deterrence or intoxication of bees (Apis mellifera) by plant allelochemicals. Chemoecology. 1993; 4(1): 8-18.

Publisher Full Text

Di Prisco G, Cavaliere V, Annoscia D, et al: Neonicotinoid clothianidin adversely affects insect immunity and promotes replication of a viral pathogen in honey bees. Proc Natl Acad Sci U S A. 2013; 110(46): 18466-71.

PubMed Abstract | Publisher Full Text | Free Full Text

Diawara MM, Trumble JT, White KK, et al:: Toxicity of linear furanocoumarins to Spodoptera exigua: Evidence for antagonistic interactions. J Chem Ecol 1993; 19(11): 2473-2484.

PubMed Abstract | Publisher Full Text

Diffenbaugh NS, Field CB: Changes in ecologically critical terrestrial climate conditions. Science. 2013; 341(6145): 486-92.

PubMed Abstract | Publisher Full Text

Dyer LA, Dodson CD, Stireman JO, et al.: Synergistic effects of three Piper amides on generalist and specialist herbivores. J Chem Ecol. 2003; 29(11): 2499-2514.

PubMed Abstract | Publisher Full Tex

Free JB: The flower constancy of bumblebees. J Anim Ecol. 1970; 39(2): 395-402.

Publisher Full Text

García-García R, López-Malo A, Palou E: Bactericidal action of binary and ternary mixtures of carvacrol, thymol, and eugenol against Listeria innocua. J Food Sci. 2011; 76(2): M95-100.

PubMed Abstract | Publisher Full Text

Gause GF, Smaragdova NP: On the killing action of optically isomeric nicotines in relation to problems of evolution of the nervous system in animals. Physiol Zool. 1939; 12(3): 238-255.

Reference Source

Gherman BI, Denner A, Bobiş O, et al.: Pathogen-associated self-medication behavior in the honeybee Apis mellifera. Behav Ecol Sociobiol. 2014; 68(11): 1777-1784.

Publisher Full Text

Goulson D, Nicholls E, Botías C, et al: Bee declines driven by combined stress from parasites, pesticides, and lack of flowers. Science. 2015; 347(6229): 1255957.

PubMed Abstract | Publisher Full Tex

Guzmán-Novoa E, Eccles L, Calvete $Y$, et al:: Varroa destructor is the main culprit for the death and reduced populations of overwintered honey bee (Apis mellifera) colonies in Ontario, Canada. Apidologie. 2010; 41(4): 443-450. Publisher Full Text

Heil M: Nectar: generation, regulation and ecological functions. Trends Plant Sci. 2011; 16(4): 191-200.

PubMed Abstract | Publisher Full Tex

Heinrich B: Temperature Regulation in the Bumblebee Bombus vagans: A Field Study. Science. 1972; 175(4018): 185-187.

PubMed Abstract | Publisher Full Text

Higes M, Martín-Hernández R, Garrido-Bailón E, et al:: Honeybee colony collapse due to Nosema ceranae in professional apiaries. Environ Microbiol Rep. 2009; 1(2): 110-113.

PubMed Abstract | Publisher Full Text

Holmstrup M, Bindesbøl AM, Oostingh GJ, et al.: Interactions between effects of environmental chemicals and natural stressors: a review. Sci Total Environ. 2010; 408(18): 3746-62.

PubMed Abstract | Publisher Full Text

Jeschke $\mathrm{P}$, Nauen $\mathrm{R}$, Schindler M, et al.: Overview of the status and global

strategy for neonicotinoids. J Agric Food Chem. 2011; 59(7): 2897-2908.

PubMed Abstract | Publisher Full Text
Kessler D, Baldwin IT: Making sense of nectar scents: the effects of nectar secondary metabolites on floral visitors of Nicotiana attenuata. Plant J. 2007; 49(5): 840-54.

PubMed Abstract | Publisher Full Text

Kluger MJ: The evolution and adaptive value of fever. Am Sci. 1978; 66(1): 38-43. PubMed Abstract

Köhler A, Pirk CW, Nicolson SW: Simultaneous stressors: interactive effects of an immune challenge and dietary toxin can be detrimental to honeybees.

$J$ Insect Physiol. 2012a; 58(7): 918-923.

PubMed Abstract | Publisher Full Text

Köhler A, Pirk CW, Nicolson SW: Honeybees and nectar nicotine: deterrence and reduced survival versus potential health benefits. $J$ Insect Physiol. 2012b; 58(2): 286-292.

PubMed Abstract | Publisher Full Tex

Kumrungsee N, Pluempanupat W, Koul O, et al.: Toxicity of essential oil compounds against diamondback moth, Plutella xylostella, and their impact on detoxification enzyme activities. J Pest Sci. 2014; 87(4): 721-729.

Publisher Full Text

Lesnoff M, Lancelot R: R package aod: Analysis of overdispersed data. 2012. Reference Source

Li HB, Zheng YT, Sun DD, et al:: Combined effects of temperature and avermectins on life history and stress response of the western flower thrips, Frankliniella occidentalis. Pestic Biochem Physiol. 2014; 108: 42-48.

PubMed Abstract | Publisher Full Text

Lipa J, Triggiani O: Crithidia bombi sp. $n$. a flagellated parasite of a bumble-bee Bombus terrestris L. (Hymenoptera, Apidae). Acta Protozoologica. 1988; 27: 287-290.

Reference Source

Lydy MJ, Belden JB, Ternes MA: Effects of temperature on the toxicity of m-parathion, chlorpyrifos, and pentachlorobenzene to Chironomus tentans. Arch Environ Contam Toxicol. 1999; 37(4): 542-547.

PubMed Abstract | Publisher Full Text

MacBean C: A world compendium: The pesticide manual. 16th edition. British Crop Production Council, Alton, UK. 2012.

Reference Source

Manson JS, Otterstatter MC, Thomson JD: Consumption of a nectar alkaloid reduces pathogen load in bumble bees. Oecologia. 2010; 162(1): 81-89. PubMed Abstract | Publisher Full Text

Manson JS, Rasmann S, Halitschke R, et al: Cardenolides in nectar may be more than a consequence of allocation to other plant parts: a phylogenetic study of Asclepias. Funct Ecol. 2012; 26(5): 1100-1110.

Publisher Full Text

Menne MJ, Durre I, Korzeniewski SM, et al.: Global Historical Climatology Network - Daily (GHCN-Daily), Version 3. NOAA National Climatic Data Center. 2012a; 3.

Publisher Full Text

Menne MJ, Durre I, Vose RS, et al:: An overview of the global historical climatology network-daily database. J Atmos Oceanic Technol. 2012b; 29: 897-910.

Publisher Full Text

Otoguro K, Iwatsuki M, Ishiyama A, et al:: In vitro antitrypanosomal activity of plant terpenes against Trypanosoma brucei. Phytochemistry. 2011; 72(16): 2024-30.

PubMed Abstract | Publisher Full Text

Otterstatter MC, Thomson JD: Within-host dynamics of an intestinal pathogen of bumble bees. Parasitology. 2006; 133(Pt 6): 749-61.

PubMed Abstract | Publisher Full Tex

Pavia CS, Pierre A, Nowakowski J: Antimicrobial activity of nicotine against a spectrum of bacterial and fungal pathogens. J Med Microbiol. 2000; 49(7): 675-6.

PubMed Abstract | Publisher Full Text

Pettis JS, vanEngelsdorp D, Johnson J, et al.: Pesticide exposure in honey bees results in increased levels of the gut pathogen Nosema. Naturwissenschaften.

results in increased

PubMed Abstract | Publisher Full Text | Free Full Text

Pinheiro J, Bates D, DebRoy S, et al.: \{nlme\}: Linear and nonlinear mixed effects models. 2015

Reference Source

Potts SG, Biesmeijer JC, Kremen C, et al.: Global pollinator declines: trends, impacts and drivers. Trends Ecol Evol. 2010; 25(6): 345-53.

PubMed Abstract | Publisher Full Text

R Core Team. R: A language and environment for statistical computing.

R Foundation for Statistical Computing, Vienna, Austria. 2014.

Reference Source

Raffa KF, Berryman AA, Simasko J, et al:: Effects of grand fir monoterpenes on the fir engraver, Scolytus ventralis (Coleoptera: Scolytidae), and its symbiotic fungus. Environ Entomol. 1985; 14(5): 552-556.

Publisher Full Text

Rattanachaikunsopon P, Phumkhachorn P: Assessment of factors influencing antimicrobial activity of carvacrol and cymene against Vibrio cholerae in food. J Biosci Bioeng. 2010; 110(5): 614-619.

PubMed Abstract | Publisher Full Text 
Richards LA, Lampert EC, Bowers MD, et al.: Synergistic effects of iridoid glycosides on the survival, development and immune response of a specialist caterpillar, Junonia coenia (Nymphalidae). J Chem Ecol. 2012; 38(10): 1276-1284.

PubMed Abstract | Publisher Full Text

Richardson LL, Adler LS, Leonard AS, et al:: Secondary metabolites in floral nectar reduce parasite infections in bumblebees. Proc Biol Sci. 2015a; 282(1803): 20142471.

PubMed Abstract | Publisher Full Text | Free Full Text

Richardson LL, Bowers MD, Irwin RE: Nectar chemistry mediates the behavior of parasitized bees: consequences for plant fitness. Ecology. 2015b.

Publisher Full Text

Salathé RM, Schmid-Hempel P: The genotypic structure of a multi-host bumblebee parasite suggests a role for ecological niche overlap. PLOS One. 2011; 6(8): e22054

PubMed Abstract | Publisher Full Text | Free Full Text

Salathé R, Tognazzo M, Schmid-Hempel R, et al.: Probing mixed-genotype infections I: extraction and cloning of infections from hosts of the trypanosomatid Crithidia bombi. PLOS One. 2012; 7(11): e49046. PubMed Abstract | Publisher Full Text | Free Full Text

Schmid-Hempel R, Eckhardt M, Goulson D, et al.: The invasion of southern South America by imported bumblebees and associated parasites. J Anim Ecol. 2014; 83(4): 823-837.

PubMed Abstract | Publisher Full Tex

Schmid-Hempel R, Tognazzo M: Molecular divergence defines two distinct lineages of Crithidia bombi (Trypanosomatidae), parasites of bumblebees. J Eukaryot Microbiol. 2010; 57(4): 337-45.

PubMed Abstract | Publisher Full Text

Schmidt TJ, Khalid SA, Romanha AJ, et al:: The potential of secondary metabolites from plants as drugs or leads against protozoan neglected diseases - part II. Curr Med Chem. 2012; 19(14): 2176-2228.

PubMed Abstract | Publisher Full Text

Simone-Finstrom MD, Spivak M: Increased resin collection after parasite challenge: a case of self-medication in honey bees? PLOS One. 2012; 7(3): e34601.

PubMed Abstract | Publisher Full Text | Free Full Text

Singaravelan N, Inbar M, Ne'eman G, et al:: The effects of nectar-nicotine on colony fitness of caged honeybees. J Chem Ecol. 2006; 32(1): 49-59.

PubMed Abstract | Publisher Full Text

Singaravelan N, Nee'man G, Inbar M, et al.: Feeding responses of free-flying honeybees to secondary compounds mimicking floral nectars. $J$ Chem Ecol. 2005; 31(12): 2791-2804

PubMed Abstract | Publisher Full Text

Singer MS, Mace KC, Bernays EA: Self-medication as adaptive plasticity: increased ingestion of plant toxins by parasitized caterpillars. PLOS One. 2009; 4(3): e4796.

PubMed Abstract | Publisher Full Text | Free Full Text

Slotsbo S, Heckmann LH, Damgaard C, et al.: Exposure to mercury reduces heat tolerance and heat hardening ability of the springtail Folsomia candida. Comp

Biochem Physiol C Toxicol Pharmacol. 2009; 150(1): 118-123.

PubMed Abstract | Publisher Full Text
Stahlschmidt ZR, Adamo SA: Context dependency and generality of fever in insects. Naturwissenschaften. 2013; 100(7): 691-696.

PubMed Abstract | Publisher Full Text

Tadmor-Melamed H, Markman S, Arieli A, et al.: Limited ability of Palestine sunbirds Nectarinia osea to cope with pyridine alkaloids in nectar of tree tobacco Nicotiana glauca. Funct Ecol. 2004; 18(6): 844-850.

Publisher Full Text

Therneau TM: R package coxme: Mixed effects cox models. 2015.

Reference Source

Thomas MB, Blanford S: Thermal biology in insect-parasite interactions. Trends Ecol Evol. 2003; 18(7): 344-350.

Publisher Full Text

Thorburn LP, Irwin RE, Adler LS, et al.: Dataset 1 in: Variable effects of nicotine and anabasine on parasitized bumble bees. F1000Research. 2015a

Data Source

Thorburn LP, Irwin RE, Adler LS, et al.: Dataset 2 in: Variable effects of nicotine and anabasine on parasitized bumble bees. F1000Research. 2015b. Data Source

Tognazzo M, Schmid-Hempel R, Schmid-Hempel P: Probing mixed-genotype infections II: high multiplicity in natural infections of the trypanosomatid, Crithidia bombi, in its host, Bombus spp. PLOS One. 2012; 7(11): e49137.

PubMed Abstract | Publisher Full Text | Free Full Text

Tomizawa M, Yamamoto I: Binding of nicotinoids and the related compounds to the insect nicotinic acetyicholine compounds receptor. J Pestic Sci. 1992; 17(4): 231-236.

Publisher Full Text

Ujváry I: Nicotine and other insecticidal alkaloids. In Yamamoto I, and Casida $\mathrm{JE}$, editors. Nicotinoid insecticides and the nicotinic acetylcholine receptor. Springer-Verlag, Tokyo. 1999: 29-69.

Publisher Full Text

Venables WN, Ripley BD: Modern applied statistics with S. Fourth edition. Springer, New York, 2002.

Publisher Full Text

Whitehead SR, Bowers MD: Chemical ecology of fruit defence: Synergistic and antagonistic interactions among amides from Piper. Funct Ecol. 2014; 28(5): 1094-1106.

Publisher Full Text

Whitehorn PR, Tinsley MC, Brown MJF, et al:: Investigating the impact of deploying commercial Bombus terrestris for crop pollination on pathogen dynamics in wild bumble bees. J Apicult Res. 2013; 52(3): 149-157.

Publisher Full Text

Williams $\mathrm{PH}$, Araújo MB, Rasmont P: Can vulnerability among British bumblebee (Bombus) species be explained by niche position and breadth? Biol Conserv. 2007; 138(3-4): 493-505

Publisher Full Text

Williams P, Colla S, Xie Z: Bumblebee vulnerability: common correlates of winners and losers across three continents. Conserv Biol. 2009; 23(4): 931-940. PubMed Abstract | Publisher Full Text

Wink M, Theile V: Alkaloid tolerance in Manduca sexta and phylogenetically related sphingids (Lepidoptera: Sphingidae). Chemoecology. 2002; 12(1): 29-46. Publisher Full Text 


\section{Open Peer Review}

\section{Current Peer Review Status: ? ?}

\section{Version 1}

Reviewer Report 19 October 2015

https://doi.org/10.5256/f1000research.7396.r10860

(C) 2015 Baracchi D. This is an open access peer review report distributed under the terms of the Creative Commons Attribution License, which permits unrestricted use, distribution, and reproduction in any medium, provided the original work is properly cited.

\section{David Baracchi}

School of Biological and Chemical Sciences, Queen Mary, University of London, London, UK

The authors investigated how the consumption of nectar alkaloids, either in isolation or combination, affect survival and pathogen load in a pollinator species. The main goal of the paper is to test the synergistic effects of two alkaloids on bee survival and parasite loads. To address this question the authors used the pollinator Bombus impatiens, its common midgut parasite, Crithidia bombi, and the natural alkaloids anabasine and nicotine, which are two nectar secondary metabolites produced by Nicotiana species. They provided evidence that both alkaloids decreased the parasite loads when bumble bees were kept under variable laboratory conditions. By contrast, they showed that nicotine but not anabasine increased the parasite load when bees were kept under controlled laboratory conditions. The consumption of the alkaloids in combinations did not have any synergistic effect, but rather an antagonistic effect especially under variable rearing conditions.

The authors concluded that the interaction between secondary metabolites are complex and that environmental variables may play an important role in determining the positive or negative impact of the diet on pollinator health.

The study addresses an important question and provides useful results. There are, however, some points that need clarification and some issues that should be addressed.

\section{Major concerns:}

In "Methods" the authors stated that " In 'Variable', pupal clumps were initially incubated on the lab bench, but were later incubated at $30^{\circ} \mathrm{C}$ in an incubator....". It is not clear if they used the bees obtained from these initial pupal clumps for the experiments or not.

If they excluded them, then there is no need to report this information. If not, authors should discuss the possible implications of this "extra" stress.

"The overall parasite loads in 'Controlled' were much higher, with median parasite loads more than double those observed in "Variable". In my view this point is very important and 
neglected by the authors and it might partially account for the apparent contradiction between the results of "Variable" and "Controlled" experiments. There are evidences for the existence of a trade-off between alkaloid toxicity to the parasite and toxicity to the host. If the parasite load is too high and bumblebee weakened, the toxicity to the bees might overcome that to the parasite, resulting in even higher parasite loads. On the other hand, if the parasite load is lower, the costs in consuming the alkaloids might have been counterbalanced by the advantages in slowing the progression of the infection. Authors should discuss this point.

Do authors have any explanation why bumble bees exposed to $2 \mathrm{ppm}$ nicotine had a so high mortality rate after only 7 days since exposure? For instance, Baracchi et al 2015 also found a toxic effect of nicotine, but the detrimental effect on bee lifespan started to be evident only about 20-30 days after the start of the experiment.

"Variable" and "Controlled" experiments also differed in time of year (the experiments were carried out in February-March and May-July respectively). Season is thus an existing confounding factor that authors should seriously take into account in the discussion.

Authors suggested that under variable conditions, bees may have consumed more liquid, causing ingestions of more alkaloids accounting for both the higher mortality and decreased parasite load in 'Variable'. Here I respectfully disagree since I would expect that bees constantly exposed to high temperatures would consume more liquid. It is also possible that bees exposed to high temperatures might have less water in the faeces or might defecate less for suppressing water loss, which may results in higher parasite load. Unfortunately, authors did not provide any information about the overall quantity of sucrose consumed by bumblebees over the experiments. I think that authors should address this point with a simple additional experiment. Measuring for few days the quantity of liquid consumed, and possibly the excretion rate of bees exposed to different temperatures, might really help them to better discuss and clarify their findings.

\section{Minor points:}

The most novel aspect of the manuscript is the fact that authors investigated the possible interactions between two natural alkaloids. I suggest to mention this aspect in the title.

In my opinion the first paragraph of the introduction is not necessary.

The second paragraph of the introduction discusses about the topic of self-medication and preferential feeding on alkaloid enriched nectars by pollinators and Baracchi et al. 2015 should be included in the paragraph since it is really pertinent here.

The authors dedicated an entire long paragraph on the chemical nature on nicotine used by Richardson et al. 2015 and the present manuscript. The paragraph is too long. On top of that, Richarson et al. 2015 and Baracchi et al 2015 (we amended this information in the paper) used different types of nicotine (the former used a +/- enantiomeric mixture, whereas the latter used (-)-enantiomer of nicotine, as the authors of the present work also did) obtaining the same results. Thus, it is improbable that the chemical composition of the alkaloid is responsible for the differences found. I suggest to reduce or remove this 


\section{paragraph.}

For the "Variable" data set authors used the GLMM while for the "Controlled" data set authors used a Cox proportional hazards mixed-effects model. Given that the "Variable" data set does not allow to use a Cox prop hazards mixed-effects model, I agree with this statistical approach, but nonetheless, it might be useful if the authors analyze the "Controlled" data also with the GLMM, so that a direct comparison of the two situations would be possible.

"Variable" and "Controlled" experiments also differed in the fact that only bees in the "Controlled" were kept with constant darkness, which could have an effect on bee locomotor activity, energy consumption etc.. Authors should mention this in the discussion.

Competing Interests: No competing interests were disclosed.

\section{I confirm that I have read this submission and believe that I have an appropriate level of expertise to confirm that it is of an acceptable scientific standard, however I have significant reservations, as outlined above.}

Author Response 07 Dec 2015

Evan Palmer-Young, University of Massachusetts at Amherst, Amherst, USA

Dear Dr. Baracchi,

Many thanks for your thorough critical review of our article. We feel that the revised version is now improved in response to your comments. We address each point below:

Reviewer comment: The study addresses an important question and provides useful results.

Author Response: We thank the reviewer for this gracious comment. Major concerns:

Reviewer Comment: In "Methods" the authors stated that "In 'Variable', pupal clumps were initially incubated on the lab bench, but were later incubated at $30^{\circ} \mathrm{C}$ in an incubator....". It is not clear if they used the bees obtained from these initial pupal clumps for the experiments or not. If they excluded them, then there is no need to report this information. If not, authors should discuss the possible implications of this "extra" stress. Author Response: We now clarify in "Methods: Rearing Conditions" that we used the bees from all of these pupal clumps. The pupal clumps were only moved to the incubator during the last week of inoculations, 2 days before the emergence of the last bees to be inoculated. Hence, all of the bees in the "Variable" experiment would have been exposed to variable temperatures during pupation. We agree that this additional stress could have affected both survival and immunity, and now mention both of these possibilities in the discussion. Reviewer Comment: "The overall parasite loads in 'Controlled' were much higher, with median parasite loads more than double those observed in "Variable". In my view this point is very important and neglected by the authors and it might partially account for the apparent contradiction between the results of "Variable" and "Controlled" experiments. There are evidences for the existence of a trade-off between alkaloid toxicity to the parasite and toxicity to the host. If the parasite load is too high and bumblebee weakened, the toxicity to the bees might overcome that to the parasite, resulting in even higher parasite 
loads. On the other hand, if the parasite load is lower, the costs in consuming the alkaloids might have been counterbalanced by the advantages in slowing the progression of the infection.

Author Response: Thank you for this suggestion. We have added the idea that alkaloids might be most medicinal against mild infections to the final paragraph of "Discussion: Interactive effects of abiotic conditions, alkaloids, and parasites on bees".

Reviewer Comment: Do authors have any explanation why bumble bees exposed to $2 \mathrm{ppm}$ nicotine had a so high mortality rate after only 7 days since exposure? For instance, Baracchi et al 2015 also found a toxic effect of nicotine, but the detrimental effect on bee lifespan started to be evident only about 20-30 days after the start of the experiment. Author Response: In the 'Variable' experiment, we believe that the stress from fluctuating temperatures contributed to overall higher levels of mortality and greater susceptibility to alkaloid toxicity than under the "standard laboratory conditions" employed in Baracchi et al (2015).

The other reviewers, Dr.'s Simone-Finstrom and Lopez-Uribe, had a similar comment about our high reported rates of mortality in 'Stable' in comparison with Richardson et al. 2015 and Baracchi et al. 2015. We duplicate that response here:

It is correct that our study found much higher rates of mortality, even under 'Stable' conditions (up to $35 \%$ over $7 \mathrm{~d}$ ), than these two prior papers. However, both of these studies employed different rearing conditions from ours in their experiments measuring survival. Richardson et al. (2015) measured mortality in microcolonies (3-bee communities) incubated in $500 \mathrm{~mL}$ plastic deli containers, as opposed to the solitary bees in $30 \mathrm{~mL}$ snap-cap vials used in our experiments. Baracchi et al. used individual bees, but they were housed in petri dishes rather than vials; in addition, whereas Baracchi et al. kept bees in the same petri dish throughout the experiment (D. Baracchi, personal communication), we moved our bees to clean vials daily, which may have imposed additional stress resulting from handling. Finally, Baracchi et al. used a different strain of Crithidia, and also a different bee species ( $B$. terrestris) from the one we used ( $B$. impatiens). Any of these social, environmental and genetic factors may have contributed to the observed differences in mortality.

Reviewer comment: "Variable" and "Controlled" experiments also differed in time of year (the experiments were carried out in February-March and May-July respectively). Season is thus an existing confounding factor that authors should seriously take into account in the discussion.

Author Response: We are hesitant to speculate on the effects of season, because bee colonies were kept indoors in cardboard boxes and inside closed cabinets, which shielded them from circadian cues. In addition, we do not know enough about the rearing conditions employed by our commercial supplier-e.g. how long bees had been raised in captivity, to what seasonal cues they were exposed-- to offer an informed discussion of this point. However, we do feel that exposure to environmental cues was an important factor within the experiment itself, and have emphasized the possible interactive effects of alkaloid consumption together with daylight and lab stimuli, as astutely pointed out in Dr. Baracchi's final comment. We mention these interactive effects in the first paragraph of "Discussion: Interactive effects of abiotic conditions, alkaloids, and parasites on bees", and reiterate their possible importance in the new Discussion paragraph contrasting our results with those of Baracchi et al.

Reviewer comment: Authors suggested that under variable conditions, bees may have consumed more liquid, causing ingestions of more alkaloids accounting for both the higher 
mortality and decreased parasite load in 'Variable'. Here I respectfully disagree since I would expect that bees constantly exposed to high temperatures would consume more liquid. It is also possible that bees exposed to high temperatures might have less water in the faeces or might defecate less for suppressing water loss, which may results in higher parasite load. Unfortunately, authors did not provide any information about the overall quantity of sucrose consumed by bumblebees over the experiments. I think that authors should address this point with a simple additional experiment. Measuring for few days the quantity of liquid consumed, and possibly the excretion rate of bees exposed to different temperatures, might really help them to better discuss and clarify their findings.

Author response: We conducted a follow-up experiment ('Consumption experiment', Figure 5) in which we measured nectar consumption at 2 different temperatures, $27^{\circ} \mathrm{C}$ and $33^{\circ} \mathrm{C}$. The lower temperature matched that used in the 'Stable' experiment (formerly 'Controlled'), whereas the higher temperature was designed to simulate typical daytime temperatures during 'Variable'. To our surprise, nectar consumption was reduced by over $50 \%$ at the higher temperature. Accordingly, we have removed speculation that bees in the generally warmer 'Variable' experiment may have consumed greater quantities of nectar and alkaloids, and instead suggest that nicotine might have exacerbated stress from dehydration.

Bees in both temperature treatments were housed in an incubator under dark conditions, similar to those in the 'Stable' experiment but lacking the light and other stimuli to which bees were exposed in the 'Variable' experiment. The interactive effects of temperature and external stimuli offer an intriguing area for further study, and we encourage such investigation in the Discussion. We thank Dr. Baracchi for suggestions and encouragement with the Consumption experiment.

Minor points:

Reviewer comment: The most novel aspect of the manuscript is the fact that authors investigated the possible interactions between two natural alkaloids. I suggest to mention this aspect in the title.

Author response: We agree. We now mention compound interactions in the title. Reviewer comment: In my opinion the first paragraph of the introduction is not necessary. Author response: This paragraph has been shortened and combined with former second paragraph.

Reviewer comment: The second paragraph of the introduction discusses about the topic of self-medication and preferential feeding on alkaloid enriched nectars by pollinators and Baracchi et al. 2015 should be included in the paragraph since it is really pertinent here. Author response: Baracchi et al. 2015 is now cited in the third paragraph of the introduction that documents medicinal effects of secondary metabolites on pollinators. Reviewer comment: The authors dedicated an entire long paragraph on the chemical nature on nicotine used by Richardson et al.2015 and the present manuscript. The paragraph is too long. On top of that, Richarson et al. 2015 and Baracchi et al 2015 (we amended this information in the paper) used different types of nicotine (the former used a +/- enantiomeric mixture, whereas the latter used (-)-enantiomer of nicotine, as the authors of the present work also did) obtaining the same results. Thus, it is improbable that the chemical composition of the alkaloid is responsible for the differences found. I suggest to reduce or remove this paragraph.

Author response: This paragraph has been reduced by half, but we felt that it was important to note this difference at some level, given the similarities between our study and 
that of Richardson et al. 2015 and the multiple studies demonstrating the disparate effects of the two enantiomers.

Reviewer comment: For the "Variable" data set authors used the GLMM while for the "Controlled" data set authors used a Cox proportional hazards mixed-effects model. Given that the "Variable" data set does not allow to use a Cox prop hazards mixed-effects model, I agree with this statistical approach, but nonetheless, it might be useful if the authors analyze the "Controlled" data also with the GLMM, so that a direct comparison of the two situations would be possible.

Author response: This analysis is now given in supplementary Table S2.

Reviewer comment: "Variable" and "Controlled" experiments also differed in the fact that only bees in the "Controlled" were kept with constant darkness, which could have an effect on bee locomotor activity, energy consumption etc.. Authors should mention this in the discussion.

Author response: We now comment on this difference in a new Discussion paragraph in which we contrast our results with those of Dr. Baracchi and colleagues.

Thank you again for the thorough review and helpful suggestions.

Sincerely,

Evan Palmer-Young on behalf of the authors

Competing Interests: I am an author on the article being refereed

Reviewer Report 07 October 2015

https://doi.org/10.5256/f1000research.7396.r10403

(c) 2015 Simone-Finstrom $M$ et al. This is an open access peer review report distributed under the terms of the Creative Commons Attribution License, which permits unrestricted use, distribution, and reproduction in any medium, provided the original work is properly cited.

\section{Michael Simone-Finstrom}

Honey Bee Breeding, Genetics, and Physiology Research, USDA Agricultural Research Service, Baton Rouge, LA, USA

\section{Margarita Lopez-Uribe}

Department of Entomology, North Carolina State University, Raleigh, NC, USA

Thorburn et al. describe an experiment where they test the dual effect of two secondary plant compounds on bumble bee mortality and parasite load after infections with the pathogen Crithidia bombi. This paper addresses an interesting question that follows up recent findings about the potential use of secondary metabolites of nectar as a self-medication mechanism to reduce parasite infections in bumble bees. However, we found several major points that the authors should address to clarify the results of the study and to better frame it within the context of previous findings.

\section{Major concerns:}

We found multiple points of the statistical analysis that were unclear: 
For the 'controlled' experiment, the authors say they used a Cox proportional hazards mixed-effect model. However, the risk ratio of death is not reported, which is one of the most informative parameters to quantitatively estimate the difference in survival between the four groups. It is also surprising that there was no significant difference between the survival of the groups. From Fig. 3, it seems that the group in the Nicotine+Anabasine had lower probability of survival.na

Wouldn't a model that allows for interactions of treatment, colony and mass be more appropriate?

Regarding mass and colony, since both terms were highly significant, how does that affect the interpretation of the results? Was there no relationship with mass and treatment? Also did the masses of bees from the 'Variable' experiment differ from those in the 'Controlled' experiment due to the stress they experienced during pupation?

The authors report a high pre-experiment mortality rate in the "controlled" treatment. Is it possible that the pupae were stressed before starting the experiment and that this condition had a significant effect in the bee survival during the experiment? Even in the control bees, a $60 \%$ mortality after 7 days was reported. This numbers seem high compared to other recent studies (Baracchi et al. 2015, Richardson et al 2015).

It was interesting to find out that the 'variable' treatment generated the most interesting results. However, the conflicting findings of the 'controlled' and 'variable treatments need to better reconcile in the discussion. There also needs to be more information reported regarding how the temperature fluctuated.

Why do authors think that the results of the 'variable treatment' were the product of heat stress and not cold stress, or just stress in general? Similarly the fact that the 'variable treatment' was during pupation and not just during the treatment period needs to be addressed along with the implications of that possible effect.

This is an overall comment about the structure of the introduction and discussion. Even though the text is well written, the structure of these sections does not connect ideas very well. It reads as a list of ideas, rather than one story with a beginning and an end.

\section{Minor comments:}

In the abstract, authors state that they tested for 'synergistic' effect between two alkaloid compounds. However, results show antagonistic effects between them. Would it be better to just broadly state that the authors are testing for possible interactions between these compounds?

The 2 nd to last paragraph in the introduction. The authors should make the point that this is the first study to look at a combination of any alkaloids, and not just nicotine and anabasine, if that's the case. It makes this study more significant and should be highlighted.

In M\&M, the subheading 'diet treatment' could be confused with a treatment where variable amounts of protein, carbohydrates and lipids were used. Would it be better to call this 
section 'Secondary Compound Treatments'?

On a similar note, the authors may consider changing the names of the treatment groups to "Variable" and "Stable" or something to that effect so there are not two control treatments.

Was the inoculation done before or after the 'diet treatment'? If it was done before, please mention this section of the methods in the order in which the experiment took place. This will help the reader with clarity about the process.

The sample sizes for the parasite load analysis could be in the text or figure, rather than in a supplementary file.

2nd paragraph of the discussion. Baracchi et al. 2015 found the same result of low levels of nicotine causing mortality, which is left out here.

The section entitled "In a controlled temperature environment..." starts by seemingly contradicting the previous section without any discussion of that. Also these results need to be reconciled with Baracchi et al. 2015.

Should be black queen cell virus (instead of brood queen cell virus)

The paragraph explaining the difference in chemical composition between the nicotine in Richardson et al and this manuscript is too long and it still doesn't explain why there was no effect on the parasite loads.

The last sentence of the discussion states that 'bees would be unlikely to consume enough nicotine from nectar to experience toxic effects'. There is a large body of literature on the effects toxic nectar on bees, how can the authors be certain about that? Particularly given that some nectar is stored within the nest and combined with other nectars.

Competing Interests: No competing interests were disclosed.

We confirm that we have read this submission and believe that we have an appropriate level of expertise to confirm that it is of an acceptable scientific standard, however we have significant reservations, as outlined above.

Author Response 07 Dec 2015

Evan Palmer-Young, University of Massachusetts at Amherst, Amherst, USA

Dear Dr.'s Simone-Finstrom and Lopez-Uribe,

Many thanks for your careful review of our research. We have revised the manuscript to incorporate your suggestions, and hope that you find the new version improved in content and clarity. We respond to each of your points below:

Major concerns:

Reviewer Comment: We found multiple points of the statistical analysis that were unclear: 
For the 'controlled' experiment, the authors say they used a Cox proportional hazards mixed-effect model. However, the risk ratio of death is not reported, which is one of the most informative parameters to quantitatively estimate the difference in survival between the four groups. It is also surprising that there was no significant difference between the survival of the groups. From Fig. 3, it seems that the group in the Nicotine+Anabasine had lower probability of survival.

Author Response: The death hazard ratios are now reported in the text, along with results of the pairwise comparison between the Control and Nicotine + Anabasine groups.

Although the Nicotine + Anabasine group did have a higher estimated death hazard ratio $(1.27 \pm 0.22 \mathrm{SE})$ compared to the control group $(0.82 \pm 0.16 \mathrm{SE})$, this difference was not statistically significant ( $p=0.446$ for pairwise comparison).

Reviewer Comment: Wouldn't a model that allows for interactions of treatment, colony and mass be more appropriate?

Author Response: We agree that it would have been interesting to investigate a more complex model. However, we were concerned that adding additional terms would prevent meaningful tests of the effects of alkaloid consumption, particularly in the 'Variable' experiment, which had a smaller sample size and an unbalanced design due to the high mortality among bees that consumed nicotine. Although the 'Stable' experiment had a larger sample size, we chose to analyze it with the same model used for the 'Variable' experiment, to facilitate comparison between the two results. Therefore, we formulated our models a priori without interaction terms. In previous studies testing the effects of secondary metabolite consumption on Crithidia infection, interaction terms were found to be non-significant (e.g. Baracchi et al. (2015)) or were not reported (e.g., Richardson et al. 2015 Proc Biol Sci. 22;282(1803):20142471. doi: 10.1098/rspb.2014.2471; Manson et al. 2010, Oecologia.62(1):81-9. doi: 10.1007/s00442-009-1431-9).

Reviewer Comment: Regarding mass and colony, since both terms were highly significant, how does that affect the interpretation of the results?

Author Response: This significance shows that Crithidia infection intensity varies by mass and also by colony, which is consistent with the results of previous studies (e.g., Otterstatter \& Thompson 2006 Parasitology 133(Pt 6):749-61.

http://doi.org/10.1017/S003118200600120X), but does not invalidate our conclusions about the effects of treatment. We have emphasized the effects of mass and colony genotype in the new paragraph of the discussion section about the differences between our study and that of Baracchi and colleagues.

Reviewer Comment: Was there no relationship with mass and treatment?

Author Response: We apologize for this confusion. Bee mass refers to mass at time of emergence from pupation, before treatments were applied. We now clarify this in Methods: Statistical analysis and in the legends of Tables 2 and 4.

Reviewer Comment: Also did the masses of bees from the 'Variable' experiment differ from those in the 'Controlled' experiment due to the stress they experienced during pupation? 
Author Response: Yes-somewhat surprisingly, bees in the 'Variable' experiment were significantly larger (mean $=0.160 \mathrm{~g}$ ) than in the 'Stable' (formerly 'Controlled') experiment $($ mean $=0.121 \mathrm{~g})$. We expect that this reflects high mortality of the smaller bees in 'Variable', which might have been more likely to die during pupation or during the two days between emergence and inoculation. This finding has been added to the section of the Results describing the 'Stable' experiment, and is mentioned again in the first paragraph of the Discussion.

Reviewer Comment: The authors report a high pre-experiment mortality rate in the "controlled" treatment. Is it possible that the pupae were stressed before starting the experiment and that this condition had a significant effect in the bee survival during the experiment?

Author Response: In the 'Variable' experiment, pre-experiment mortality was indeed high, and yes, likely impacted survival during the experiment as well. The chronic exposure of colonies, pupae, and adult bees to variable temperatures is now noted in the third paragraph of "Methods: Rearing conditions" and in the first paragraph of the discussion. Reviewer Comment: Even in the control bees, a $60 \%$ mortality after 7 days was reported. This numbers seem high compared to other recent studies (Baracchi et al. 2015, Richardson et al 2015).

Author Response: It is correct that our study found much higher rates of mortality, even under 'Stable' conditions (up to 35\% over $7 \mathrm{~d}$ ), than these two prior papers. However, both of these studies employed different rearing conditions from ours in their experiments measuring survival. Richardson et al. (2015) measured mortality in microcolonies (3-bee communities) incubated in $500 \mathrm{~mL}$ plastic deli containers, as opposed to the solitary bees in $30 \mathrm{~mL}$ snap-cap vials used in our experiments. Baracchi et al. used individual bees, but they were housed in petri dishes rather than vials; in addition, whereas Baracchi et al. kept bees in the same petri dish throughout the experiment (D. Baracchi, personal communication), we moved our bees to clean vials daily, which may have imposed additional stress resulting from handling. Finally, Baracchi et al. used a different strain of Crithidia, and also a different bee species ( $B$. terrestris) from the one we used (B. impatiens). Any of these social, environmental and genetic factors may have contributed to the observed differences in mortality. These differences are described in the new Discussion paragraph contrasting our study with that of Baracchi et al.

Reviewer Comment: It was interesting to find out that the 'variable' treatment generated the most interesting results. However, the conflicting findings of the 'controlled' and 'variable treatments need to better reconcile in the discussion.

Author Response: We have added and clarified speculation about the reasons for these differences and their implications in "Discussion: Interactive effects of abiotic conditions, alkaloids, and parasites on bees", wherein we consider a number of non-exclusive possible explanations for differences in the effects of alkaloids' effects on mortality and parasitism between 'Variable' and 'Stable':

(1) Increased consumption: We hypothesized that the stronger effects of alkaloids in 'Variable' may have resulted from increased liquid (nectar) intake under hot conditions. We tested this idea in the 'Consumption experiment'. To our surprise, bees drank substantially less at high temperatures similar to those in 'Variable'. These results are now reported (Figure 5, Table 5).

(2) Interactions between multiple stressors:

(a) Mortality: Stronger effects of alkaloid intake on mortality in 'Variable' may reflect 
increased susceptibility to alkaloid toxicity in the presence of multiple stressors, including temperature fluctuation and external disturbance. Given the results of the 'Consumption experiment', we now speculate that nicotine may have increased mortality in 'Variable' by increasing fecal moisture losses. Because the warmer temperatures of 'Variable' appear to have increased evaporative moisture loss while reducing nectar intake, any additional nicotine-induced moisture loss would have increased the risk of lethal dehydration.

(b) Parasitism: By the same logic, stronger effects of alkaloid intake on parasitism in variable temperatures may reflect synergistic effects of alkaloids and external stimuli on locomotor and gut activity. In the new paragraph describing the differences between our study and that of Baracchi et al. (2015), we have noted that we both we and Baracchi et al. showed antiparasitic effects of nicotine consumption when bees were kept on the lab bench (as they were in 'Variable'), but not when bees were housed in constant darkness (as in 'Stable'). (3) Inhibitory effects of high temperatures on Crithidia compensating for nicotine-induced immunosuppression: We suggest that in 'Stable', immunosuppressive effects of nicotine contributed to increased parasite loads. In contrast, in 'Variable', stimulatory effects of alkaloid consumption on locomotor activity may have caused bee body temperature to reach or exceed the thermal tolerance limits of the parasite. These febrile effects may have offset any immunosuppressive effects of nicotine.

(4) In the paragraph describing differences between Crithidia strains, we have incorporated an additional suggestion from the other reviewer. Dr. Baracchi suggested that nicotine and anabasine may have their greatest medicinal value at lower levels of infection, such as those observed in 'Variable', where median Crithidia counts were lower than in 'Stable'.

Reviewer Comment: There also needs to be more information reported regarding how the temperature fluctuated. Why do authors think that the results of the 'variable treatment' were the product of heat stress and not cold stress, or just stress in general?

Author Response: We agree that the effects seen in 'Variable' could reflect both heat and cold stress. However, my personal recollection (ECPY) was that most of the time the lab was very hot, around $35^{\circ} \mathrm{C}$, at least during the day when the experimenters were present. Cold stress seemed to be more occasional, with sporadic morning and weekend periods when the temperature decreased to $10-15^{\circ} \mathrm{C}$ due to building steam being shut off for repairs. We would have liked to have kept more careful records, ideally from data loggers, but the extent and duration of the variability in temperature was entirely unexpected, and we did not consistently note the precise conditions. Additional detail about the temperature fluctuation has been added to the first paragraph of Methods: Rearing conditions.

Reviewer Comment: Similarly the fact that the 'variable treatment' was during pupation and not just during the treatment period needs to be addressed along with the implications of that possible effect.

Author Response: We have now noted this additional stress throughout the developmental period in 3 places: in the third paragraph of "Methods: Rearing conditions", at the end of "Results: 'Stable"', and in the first paragraph of the discussion.

Reviewer Comment: This is an overall comment about the structure of the introduction and discussion. Even though the text is well written, the structure of these sections does not connect ideas very well. It reads as a list of ideas, rather than one story with a beginning and an end.

Author Response: We appreciate this gracious compliment, and have attempted to improve the organization of the introduction, where we have shortened or separated some paragraphs. In the discussion, we have shortened the paragraph contrasting our results 
with those of Richardson et al. (2015), and have elaborated on the differences between 'Stable' and 'Variable' as well as between our study and previous ones.

\section{Minor comments:}

Reviewer Comment: In the abstract, authors state that they tested for 'synergistic' effect between two alkaloid compounds. However, results show antagonistic effects between them. Would it be better to just broadly state that the authors are testing for possible interactions between these compounds?

Author Response: We agree and have rephrased the abstract.

Reviewer Comment: The 2nd to last paragraph in the introduction. The authors should make the point that this is the first study to look at a combination of any alkaloids, and not just nicotine and anabasine, if that's the case. It makes this study more significant and should be highlighted.

Author Response: Thank you. We now highlight this unique contribution in the aforementioned paragraph.

Reviewer Comment: In M(aterials\&Methods), the subheading 'diet treatment' could be confused with a treatment where variable amounts of protein, carbohydrates and lipids were used. Would it be better to call this section 'Secondary Compound Treatments'?

Author Response: We have implemented this suggestion.

Reviewer Comment: On a similar note, the authors may consider changing the names of the treatment groups to "Variable" and "Stable" or something to that effect so there are not two control treatments.

Author Response: We apologize that the naming of the "Controlled" experiment was confusing. We have implemented this suggestion and renamed the "Controlled" experiment to "Stable".

Reviewer Comment: Was the inoculation done before or after the 'diet treatment'? If it was done before, please mention this section of the methods in the order in which the experiment took place. This will help the reader with clarity about the process.

Author Response: We apologize for the confusion. The inoculation was performed before the secondary compound treatment began. Bees were fed $30 \%$ sucrose nectar without secondary metabolites for 2 days after emergence, then inoculated with Crithidia. Diet treatments were implemented immediately post-inoculation. We have reordered this description in the methods section ("Rearing conditions": final paragraph).

Reviewer Comment: The sample sizes for the parasite load analysis could be in the text or figure, rather than in a supplementary file.

Author response: We have now listed the sample sizes for each analysis in the legends of the relevant figures.

Reviewer Comment: 2nd paragraph of the discussion. Baracchi et al. 2015 found the same result of low levels of nicotine causing mortality, which is left out here.

Author response: This result is now cited (third paragraph of Discussion).

Reviewer Comment: The section entitled "In a controlled temperature environment..." starts by seemingly contradicting the previous section without any discussion of that.

Author response: We have added two transition sentences at the beginning of this paragraph.

Reviewer Comment: Also these results need to be reconciled with Baracchi et al. 2015.

Author response: We have added a full paragraph to the discussion section outlining the differences between our experiments and those of Baracchi et al. (2015). 
Reviewer Comment: Should be black queen cell virus (instead of brood queen cell virus) Author response: Thank you for pointing out this error. It is now corrected. Reviewer Comment: The paragraph explaining the difference in chemical composition between the nicotine in Richardson et al and this manuscript is too long and it still doesn't explain why there was no effect on the parasite loads.

Author response: This paragraph has been substantially reduced, but is retained in a shortened form; the editors encouraged us to include this paragraph in order to reconcile our results with those of Richardson et al., which included experiments done in our own laboratory.

Reviewer Comment: The last sentence of the discussion states that 'bees would be unlikely to consume enough nicotine from nectar to experience toxic effects'. There is a large body of literature on the effects toxic nectar on bees, how can the authors be certain about that? Particularly given that some nectar is stored within the nest and combined with other nectars.

Author response: We have now reduced this speculation to say that in varied landscapes, bees might be able to reduce or avoid toxicity by exploiting different, lower-nicotine flower species.

Thanks you again for your critical review and thoughtful suggestions.

Sincerely,

Evan Palmer-Young on behalf of the authors

Competing Interests: I am an author of the manuscript being reviewed

The benefits of publishing with F1000Research:

- Your article is published within days, with no editorial bias

- You can publish traditional articles, null/negative results, case reports, data notes and more

- The peer review process is transparent and collaborative

- Your article is indexed in PubMed after passing peer review

- Dedicated customer support at every stage

For pre-submission enquiries, contact research@f1000.com 